\title{
TGF beta Exposure Promotes Acquisition of Epithelial-like Phenotype in Breast Cancer Spheres
}

\author{
Xin $\mathrm{Yu}^{1}$, Meizhen $\mathrm{Yin}^{2}$, Zhenhong $\mathrm{Su}^{2}$ and Jie Huang ${ }^{1^{*}}$ \\ ${ }^{1}$ Hubei Key Laboratory of Kidney Disease Pathogenesis and Intervention, School of Medicine, Hubei Polytechnic University, Huangshi 435003, PR China \\ ${ }^{2}$ School of Medicine, Hubei Polytechnic University, Huangshi 435003, PR China
}

"Corresponding author: Jie Huang, Hubei Key Laboratory of Kidney Disease Pathogenesis and Intervention, School of Medicine, Hubei Polytechnic University, 16 Guilin North Road, Huangshi, Hubei 435003, PR China, Tel: 86-27-88039076; E-mail: doctor.jie.huang@gmail.com

Rec date: May 12, 2015; Acc date: Jun 01, 2015; Pub date: Jun 03, 2015

Copyright: (c) $2015 \mathrm{Yu} \mathrm{X}$, et al. This is an open-access article distributed under the terms of the Creative Commons Attribution License, which permits unrestricted use, distribution, and reproduction in any medium, provided the original author and source are credited.

\begin{abstract}
The epithelial-to-mesenchymal transition (EMT) is considered as a critical developmental process in cancer biology. TGF beta has been shown to play an important role in the induction of EMT via down-regulating E-cadherin and up-regulating vimentin expression in cancer cells. However, whether TGF beta induces EMT in cancer spheres has not been determined yet. To address this question, we generated breast cancer spheres from MCF7 and MDAMB-231 breast cancer cell lines. These cancer spheres then were treated with recombinant human TGF beta for 24 and 48 hours, respectively in order to examine the expression of epithelial marker E-cadherin and mesenchymal marker vimentin. We find that breast cancer spheres enriched with E-cadherin and exhibit characteristics of epithelial cells by TGF beta stimulation. In addition, we did not observe TGF beta induces EMT in breast cancer spheres such as lose E-cadherin expression and gain vimentin expression. Our data suggested that TGF beta plays a different role in breast cancer spheres and cancer cells during EMT induction.
\end{abstract}

Keywords Breast cancer spheres; TGF beta; EMT

\section{Introduction}

The epithelial-to-mesenchymal transition (EMT) is a process by which cells undergo a morphological change from the epithelial polarized phenotype to a mesenchymal phenotype [1]. In this process, cells lose their epithelial features and gain mesenchymal properties to become invasive. The feature of EMT is the reduction of E-cadherin which is critical to maintain the epithelial structure [2]. It has been reported that loss of E-cadherin expression is correlated with tumor invasion and metastasis [3]. With the loss of E-cadherin expression, the expression of mesenchymal markers such as vimentin can be upregulated when EMT occurs [4]. These processes may promote the progression and invasion of cancer cell into the surrounding microenvironment. Transforming growth-factor (TGF) beta is an important inducer of EMT [5]. TGF beta-induced EMT often promotes loss of E-cadherin expression [6]. In early tumorigenesis, TGF beta inhibits the growth of epithelial cells, in contrast to the growth inhibitory effects of TGF beta in the early stages of carcinogenesis, TGF- $\beta 1$ can also act as a promoter of tumor cell invasion and metastasis in the later stages of tumorigenesis [7]. Therefore, TGF beta-dependent EMT in cancer cells can be mediated by the ability of TGF beta to induce the expression of E-cadherin gene repressors as well as its ability to alter the cell junctions. In addition, TGF beta has been described to induce EMT and an enhanced metastatic potential in breast cancer cells, MCF7 and MDA-MB-231 [8].

Recently, sphere culture has been used as a method for enriching stem cells. Scientists have reported the application of sphere culture to isolate, enrich, maintain or expand potential cancer stem cell subpopulations from various types of cancers [9]. The sphere-forming cells from primary breast cancer showed stem-like properties and expressed their cancer stem cell markers [10]. Generally, it is agreed that cancer sphere-forming cells are capable of proliferation, selfrenewal and possess higher tumorigenicity.

However whether TGF-beta induces EMT in breast cancer spheres has not been investigated yet.

In this study, we investigated the ability of the TGF beta stimulation in inducing EMT in breast cancer spheres by using immunofluorescent staining. After TGF-beta treatment, EMT in breast cancer spheres was examined in the expression of key molecules such as CK18, estrogen receptor (ER), E-cadherin, and vimentin. Morphologic changes of the breast cancer spheres were then evaluated in Zeiss LSM510 confocal laser-scanning microscope.

\section{Materials and Methods}

\section{Antibodies and reagents}

Recombinant human TGF beta was obtained from Sigma. CK18 antibody was obtained from Cell Signaling Technology. E-Cadherin antibody was obtained from BD Transduction Laboratories. Estrogen receptor antibody was obtained from Santa Cruz Biotechnology. Vimentin antibody was obtained from Chemicon.

\section{Breast cancer cells culture}

The breast cancer cell lines, MCF7 and MDA-MB-231 were obtained from American Type Culture Collection. Cancer cell lines were grown in Dulbecco's Modified Eagle Medium (DMEM) (HyClone, Logan, UT) supplemented with $10 \%$ fetal bovine serum (FBS) (Gemini Bio-Products, Woodland, CA) and 1\% penicillin/ streptomycin solution (Thermo Fisher Scientific, Waltham, MA). Cancer cells were incubated at $37^{\circ} \mathrm{C}$ in a humidified incubator with $5 \%$ $\mathrm{CO}_{2}$. 


\section{Generation of breast cancer spheres}

MCF7 and MDA-MB-231 cancer cells were suspended at $1 \mathrm{X} 105$ cells $/ \mathrm{ml}$ and seeded into ultralow attachment plates (Corning, NY) in serum free DMEM/F12 (1:1) supplemented with $20 \mathrm{ng} / \mathrm{ml}$ epidermal growth factor (EGF, peprotech) and ITS (insulin+transferring +selenium, Sigma), with or without B27 (GIBCO). $2 \mathrm{ml}$ of fresh media was added to each well every 3 days. Cancer cells grown in these conditions as non-adherent spherical clusters of cells (cancer spheres) were collected every 7 days by gentle centrifugation and dissociated to single cell suspensions.

\section{Immunofluorescent staining}

Breast cancer spheres were collected by centrifugation in a Cytospin (Thermo, Waltham, MA) onto glass slides prior to fixation. For monolayer cultures, breast cancer spheres were grown on LAB-TEK II chamber slides (Nalgene Nunc International, Penfield, NY). Breast cancer spheres were fixed in $4 \%$ paraformaldehyde for $15-30 \mathrm{~min}$ at room temperature, washed with PBS and permeabilized/blocked for $1 \mathrm{~h}$ at room temperature with PBS, $0.3 \%$ Triton X-100, 2.5\% horse serum. Slides were then incubated with anti-CK18, anti-ER, anti-Ecadherin, and anti-vimentin primary antibodies (diluted 1:100) overnight, at $4^{\circ} \mathrm{C}$ washed in PBS. Alex 488 and 568 secondary antibodies were added at a dilution of 1/200 and incubated for $1 \mathrm{~h}$ at room temperature, and after washing in PBS, DAPI dye was added to reveal nuclear DNA. Immunofluorescence was visualized in a Zeiss LSM510 confocal laser-scanning microscope.

\section{Results}

\section{Expression of CK18 and ER in breast cancer spheres}

Upon treatment with TGF beta during 24 and 48 hours, no significant change in expression of estrogen receptor was detectable in treated MCF7 and MDA-MB-231 breast cancer spheres compared to untreated cancer spheres (Figure 1). In addition, immunofluorescence analysis indicated that ER positive staining was detected in MCF7 cancer spheres while ER negative staining was detectable in MDAMB-231 cancer spheres. Regarding cytokeratin CK18 expression, before and after treatment it was strongly expressed in all cancer spheres. In particular, CK18 was localized in the border of each cancer sphere, but there was no significant difference between those untreated and treated with TGF beta for 24 and 48 hours. These results suggest that TGF beta stimulation did not change primary features of breast cancer spheres.

\section{Expression of E-cadherin and Vimentin in breast cancer spheres}

The effect of TGF beta treatment in breast cancer cell lines suggested that TGF beta promoted an EMT. The morphological changes of breast cancer cells undergoing EMT are accompanied by a shift in expression from an epithelial to a mesenchymal repertoire. To determine whether TGF beta also induces such shift in breast cancer spheres, we used immunofluorescence staining to examine the expression of E-cadherin and vimentin. Comparing to the untreated cancer spheres, E-cadherin was significantly overexpressed in treated breast cancer spheres with TGF beta for 24 and 48 hours in both MCF7 and MDA-MB-231 breast cancer spheres. Interestingly, there was no significant change in vimentin expression even though vimentin was strongly expressed in untreated and treated cancer spheres. In all untreated spheres, E-cadherin was expressed in the border of each cancer spheres. After treatment, E-cadherin was uniformly expressed in the whole spheres (Figure 2). These results suggest that TGF beta played a different role in between breast cancer spheres and breast cancer cell lines.

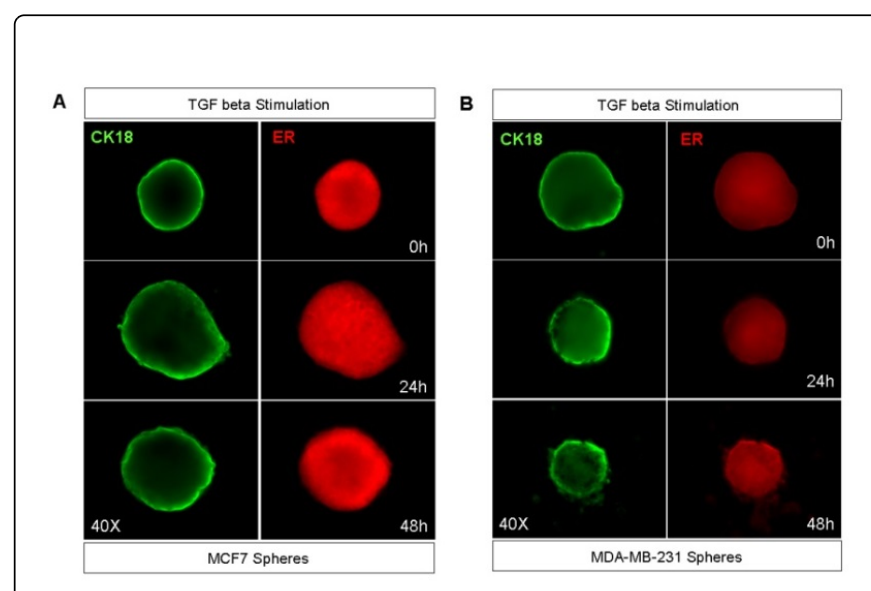

Figure 1: Immunofluorescence analyses of CK18 and ER antibodies in breast cancer spheres from MCF7 and MDA-MB-231 cancer cell lines. MCF7 (A) and MDA-MB-231 (B) breast cancer spheres were stained using CK18 antibody, a breast luminal epithelial cell marker. Strong staining of CK18 was detected in breast cancer spheres of untreated and treated with TGF beta during 24 and 48 hours. Whereas the positive staining of ER, a hormone-dependent breast cancer marker was only restricted in MCF7 cancer spheres (A) of untreated and treated with TGF beta during 24 and 48 hours. TGF beta stimulation did not change the expression of CK18 and ER in both MCF7 (A) and MDA-MB-231 (B) breast cancer spheres. CK18 was stained with Alexa 488 green labeled and ER was stained with Alexa 568 red labeled secondary antibodies. Original magnification: $400 \mathrm{X}$.

\section{Discussion}

The questions we tried to answer in this study are: does TGF beta work on all different sphere subpopulations or only on specific sphere subpopulations? Does TGF beta induce E-cadherin expression and is this effect dependent on the timing of TGF beta stimulation?

For this purpose, we used two breast cancer cell lines, MCF7 (ER positive) and MDA-MB 231 (ER negative) to obtain breast cancer spheres. Breast cancer cells are plated at a low density $(<5000$ cells/well in 6-well plate) to avoid spontaneous cell aggregation. The two cell lines could form clonal non-adherent 3-D spheres, and could also be serially passaged. After treatment with TGF beta, both breast cancer spheres became more epithelial-like phenotype compared to the untreated spheres. In our study, we surprisingly observed that Ecadherin shows a stronger expression in the border of spheres in the untreated cancer spheres while most E-cadherin expresses in the whole spheres in the treated cancer spheres after TGF beta treatment. ER +MCF7 and ER-MDA-MB 231 cancer spheres showed a major efficiency in expression of E-cadherin compared to the corresponding untreated cancer spheres, whereas ER+MCF7 and ER-MDA-MB 231 cancer spheres were not changes in the expression of vimentin compared to untreated cancer spheres. 
Citation: Yu X, Yin M, Su Z, Huang J (2015) TGF beta Exposure Promotes Acquisition of Epithelial-like Phenotype in Breast Cancer Spheres. J Cytol Histol S3: 014. doi:10.4172/2157-7099.S3-014

Page 3 of 3

A
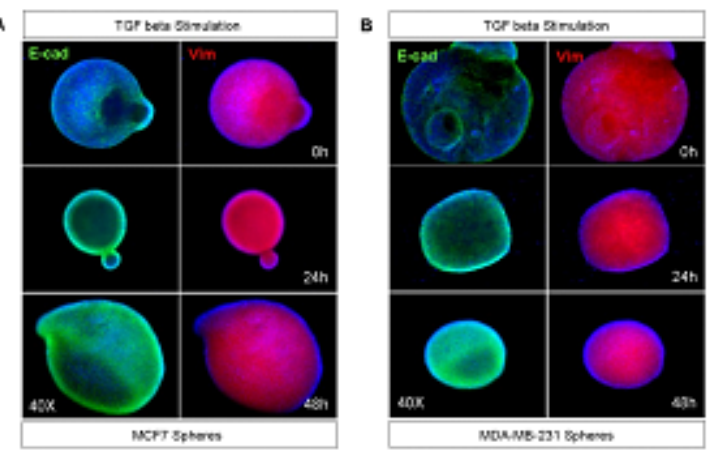

Figure 2: Immunofluorescence analyses of E-cadherin and vimentin antibodies in breast cancer spheres from MCF7 and MDA-MB-231 cancer cell lines. MCF7 (A) and MDA-MB-231 (B) breast cancer spheres were stained using E-cadherin (E-cad) antibody, an epithelial marker. The expression of E-cadherin was significantly increased in MCF7 (A) and MDA-MB-231 (B) breast cancer spheres after treatment of TGF beta for 24 and 48 hours, compared to untreated breast cancer spheres. The positive staining of vimentin, a mesenchymal marker, was detected in both MCF7 and MDA-MB0231 breast cancer spheres. However, the expression of vimentin was not changed in breast cancer spheres in between untreated and treated with TGF beta during 24 and 48 hours. Ecadherin was stained with Alexa 488 green labeled and vimentin was stained with Alexa 568 red labeled secondary antibodies. Nuclei were stained with $4^{\prime}, 6^{\prime}$-diamidino-2-phenylindole (DAPI) (blue). Original magnification: 400X.

In this study, our data indicate that MCF7 and MDA-MB-231 breast cancer spheres did not undergo EMT in response to TGF beta stimulation during 24 and 48 hours. Interestingly, the expression of Ecadherin was significantly increased after TGF beta treatment with 24 and 48 hours, while vimentin expression was not change and can be strongly detected in untreated and treated cancer spheres. In addition, we investigated the role of TGF beta in different sphere subpopulations in order to identify the sphere subset responding to TGF beta treatment.

In summary, our study demonstrated that the TGF beta induces the overexpression of E-cadherin in breast cancer spheres from MCF7 and MDA-MB-231 breast cancer cell lines, which have a different feature of EMT comparing to TGF beta-induced EMT in breast cancer cell lines. The mechanisms of the induction of EMT by TGF beta in breast cancer spheres remain to be investigated.

\section{Author's contributions}

Huang J conceived the study and participated in the study design, coordination and manuscript writing. Xin Yu participated in the study performance. Meizhen Yin and Zhenhong Su participated in the study performance and coordination. All authors have read and approved the final manuscript.

\section{Acknowledgements}

This study was supported by the Funding of Hubei Key Laboratory of Kidney Disease Pathogenesis and Intervention.

\section{References}

1. Kalluri R, Weinberg RA (2009) The basics of epithelial-mesenchymal transition. J Clin Invest 119: 1420-1428.

2. Lee JM, Dedhar S, Kalluri R, Thompson EW (2006) The epithelialmesenchymal transition: new insights in signaling, development, and disease. J Cell Biol 172: 973-981.

3. Cavallaro U, Christofori G (2001) Cell adhesion in tumor invasion and metastasis: loss of the glue is not enough. Biochem Biophys Acta 1552: $39-45$.

4. Onder TT, Gupta PB, Mani SA, Yang J, Lander ES, et al. (2008) Loss of Ecadherin promotes metastasis via multiple downstream transcriptional pathways. Cancer Res 68: 3645-3654.

5. Miyazono K (2009) Transforming growth factor-beta signaling in epithelial-mesenchymal transition and progression of cancer. Proc Jpn Acad Ser B Phys Biol Sci 85: 314-323.

6. Xu J, Lamouille S, Derynck R (2009) TGF-beta-induced epithelial to mesenchymal transition. Cell Res 19: 156-172.

7. Jakowlew SB (2006) Transforming growth factor-beta in cancer and metastasis. Cancer Metastasis Rev 25: 435-457.

8. Tomaskovic-Crook E, Thompson EW, Thiery JP (2009) Epithelial to mesenchymal transition and breast cancer. Breast Cancer Res 11: 213.

9. Miettinen PJ, Ebner R, Lopez AR, Derynck R (1994) TGF-beta induced transdifferentiation of mammary epithelial cells to mesenchymal cells: involvement of type I receptors. J Cell Biol 127: 2021-2036.

10. Oft M, Peli J, Rudaz C, Schwarz H, Beug H, et al. (1996) TGF-betal and Ha-Ras collaborate in modulating the phenotypic plasticity and invasiveness of epithelial tumor cells. Genes Dev 10: 2462-2477.
This article was originally published in a special issue, entitled:

"Cytopathology", Edited by Borislav A. Alexiev, University of Maryland Medical Center, USA 